\title{
CORRIGENDUM
}

\section{COMPLEX BERWALD MANIFOLDS WITH VANISHING HOLOMORPHIC SECTIONAL CURVATURE - CORRIGENDUM}

\section{By RONGMU YAN}

School of Mathematical Science, Xiamen university, 361005, P.R. China

e-mail: yanrm@xmu.edu.cn

doi:10.1017/S001708950800414X, Published by Glasgow Mathematical Journal Trust 21 April 2008.

In [1], we consider the class of Kähler-Finsler metrics $F$ on a complex manifold $M$ which are strongly convex. We claimd that a complex Finsler metric of this kind is complex Berwald and with vanishing holomorphic sectional curvature if and only if it is complex locally Minkowski. Unfortunately, the proof of the 'only if' part was incorrect, since we assumed that the 2 -form $\Omega_{\beta}^{\alpha}$ is 0 , claiming erroneously that this was a consequence of the vanishing of the holomorphic sectional curvature (see line 5 at p. 208). However, all other arguments remain correct if the hypothesis on the holomorphic sectional curvature is replaced by the slightly stronger condition $\Omega_{\beta}^{\alpha}=0$. In other words, the main theorem in [1] should be corrected as follows:

MAIN THEOREM. Let $F$ be a strongly convex and Kähler-Finsler metric on a complex manifold $M$. Then it is a complex Berwald metric with curvature 2-form $\Omega_{\beta}^{\alpha}=0$ if and only if it is a complex Minkowski metric.

We remark that the condition $\Omega_{\beta}^{\alpha}=0$ is true whenever the complex Finsler metric satisfies a certain natural condition on the torsion. A discussion of this fact and a generalization of the above theorem will be presented elsewhere.

\section{REFERENCE}

1. R. Yan, Complex Berwald manifolds with vanishing holomorphic sectional curvature, Glasgow Math. J. 50 (2008), 203-208.

*Supported by the National Natural Science Foundation of China (No. 10771174). 